\title{
Implicancias tributarias del convenio de la Comunidad Andina de Naciones -CAN- en los servicios de consultoría empresarial de Perú a Colombia ${ }^{1}$
}

\author{
Liliana Rojas Silva ${ }^{2}$ \\ Alessandro Martín Rodríguez Chumpitaz ${ }^{3}$
}

Recibido: 6 de julio de 2016

Aprobado: 12 de agosto de 2016

\begin{abstract}
Rojas, L., y Rodríguez, A. (2016). Implicancias tributarias del convenio de la Comunidad Andina de Naciones -CAN- en los servicios de consultoría empresarial de Perú a Colombia. Activos, 27, 61-76.
\end{abstract}

Clasificación JEL: H25, F53

\section{Resumen}

El presente documento explica las implicancias tributarias generadas por la aplicación del convenio de la Comunidad Andina de Naciones (en adelante CAN) para evitar los problemas derivados de la doble imposición tributaria (entendido como el pago de tributos de la misma renta en dos estados)

1 Documento presentado al XI Congreso Nacional y I Internacional de Estudiantes de Contaduría Pública, Universidad Santo Tomás, celebrado en dicha universidad, los días 6 y 7 de mayo de 2016 en la ciudad de Bogotá, Colombia.

2 Estudiante de Contaduría de la Universidad Nacional Mayor de San Marcos, Facultad de Ciencias Contables. Correo electrónico: lily_unmsm_14@hotmail.com

3 Estudiante de Contaduría de la Universidad Nacional Mayor de San Marcos, Facultad de Ciencias Contables. Correo electrónico: odriguezchumpitaz@gmail.com 
en la prestación de servicios de consultoría contable, se analiza el caso de una consultoría empresarial de una empresa peruana a una colombiana realizada en el año 2015. La consultoría es un tipo de relación de ayuda establecida entre diferentes actores -el consultor y la organización- basada por un lado sobre los conocimientos, las habilidades y las acciones del consultor, y por otro sobre el conocimiento, la colaboración y la necesidad de la empresa-cliente.

El trabajo identifica y analiza los beneficios y perjuicios económicos empresariales generados por la aplicación del convenio de la CAN en la prestación de servicios de consultoría, especialmente sobre los rubros ingresos exonerados del Impuesto de Renta, su retención y los gastos vinculados al servicio prestado.

\title{
Palabras clave
}

Comunidad Andina de Naciones, tributación, renta de fuente extranjera, servicios de consultoría empresarial.

\section{Rojas, L., y Rodríguez, A. (2016). Tax impliances of Andean Nations Community Agreement -ANCA- in the business consul- ting services from Peru to Colombia. Activos, 27, 61-76.}

\begin{abstract}
This document explain the tax implicancies caused by application of Andean Nations Community Agreement (onwards ANCA) with the purpose to prevent the derivated problems of double taxation in provision of services of accounting advisor, it analyses a case of a business consulting from a peruvian company to a colombian Company realized in 2015.

This paper identify and analyses the economic benefits and damages generated by application of ANCA in offer of consulting services, specially on
\end{abstract}


the exonerated incomes of Income Tax, the retentions of Income Tax and the expenses vinculated to services provisions.

\section{Keywords}

Andean Nations Community, taxation, foriegn source income, consulting business services.

Rojas, L., y Rodríguez, A. (2016). Implications fiscales de l'accord de la Communauté Andine des Nations -CAN- sur les services de conseil aux entreprises du Pérou à la Colombie. Activos, 27, 61-76.

\section{Résumé}

Ce document explique les implications fiscales générées par la mise en œuvre de l'accord de la Communauté Andine des Nations (CAN) pour éviter les problèmes liés à la double imposition (considéré comme le paiement des impôts sur le même revenu dans deux états) pour les expertises comptables; on analyse le cas d'une expertise comptable d'une entreprise péruvienne à une entreprise colombienne menée en 2015. Le service d'expertise est un genre de relation d'aide établie entre les différents acteurs -le consultant et l'entreprise- basée d'une part sur les connaissances, les compétences et les actions du consultant, et de l'autre sur la connaissance, la collaboration et le besoin de l'entreprise-cliente.

Ce travail identifie et analyse les avantages et les pertes économiques commerciales générées par l’application de l'accord de la CAN dans le domaine des services de conseil, en particulier sur les revenus exonérés de l'impôt sur le revenu, les charges de rétention et les dépenses liées au travail au service fourni.

\section{Mots-clés}

Communauté Andine des Nations, fiscalité, revenus de source étrangère, services de conseil aux entreprises. 


\section{Introducción}

El presente documento apunta a identificar y analizar las implicancias tributarias en el Impuesto de Renta -IR- que se generan al aplicar el convenio de la CAN en los servicios de consultoría contable prestados de Perú a Colombia en el periodo 2015. Asimismo, el impacto positivo y negativo del convenio que influenciarán en la rentabilidad económica de las empresas involucradas.

Parte de plantearse la pregunta sobre las implicancias tributarias de la aplicación del convenio de la CAN en los servicios de consultoría contable prestados por Perú a Colombia en el periodo 2015, tanto beneficiosas como perjudiciales para las partes que se involucran en el proceso de consultoría, y particularmente entre empresas peruanas y colombianas.

El documento usa una metodología de tipo descriptivo y analítico en el que se busca analizar la aplicación del convenio de la CAN en el tratamiento tributario del IR entre países miembros. Mediante este método se recopilan diversos informes y resoluciones realizados por las Administraciones Tributarias de Perú y Colombia, informes preparados por la CAN y documentos que analizan la prestación de servicios de consultoría contable por parte de empresas peruanas, con el fin de analizar los beneficios y perjuicios económicos generados para los prestadores en el marco de este acuerdo.

El documento parte de identificar que el principal problema en el comercio de servicios de consultoría en el marco del convenio CAN, lleva a que las empresas peruanas, prestadoras de servicios, deberán reconocer sus ingresos como exonerados y deducibles para efectos del IR, y será objeto de retención en la fuente del IR correspondiente, el cual no podrá ser usado ni como crédito fiscal ni como gasto para efecto de la declaración de dicho impuesto, e igualmente los gastos directamente relacionados al servicio no serán deducibles.

Esto porque en concordancia con el artículo n. ${ }^{\circ} 14$ de la decisión n. ${ }^{\circ} 578$ del convenio de la CAN, las rentas obtenidas producto de la prestación de 
servicios de consultoría serán gravables solo en un país miembro, evitando así la doble imposición, lo cual generaría un beneficio económico para las empresas. Sin embargo, al mismo tiempo la empresa peruana tendrá que incurrir en gastos y costos vinculados al servicio que no serán deducibles para efectos del IR, tal como establece el inciso p) del artículo n. ${ }^{\circ} 21$ del Reglamento de la Ley del IR (legislación peruana), lo cual generaría un perjuicio económico a los prestadores.

\section{Marco teórico}

\section{Antecedentes}

Según Gálvez (2010, p. 1), el análisis de las implicaciones tributarias derivadas de las transacciones de venta de servicios de empresas peruanas al exterior, viene adquiriendo una mayor relevancia en la medida en que las grandes empresas de capital peruano comienzan a invertir con mayor fuerza en el extranjero, y tienen unos mayores niveles de intercambio. Estas empresas prestan diversos servicios de consultoría empresarial (contable y administrativo principalmente), generando ingresos de fuente extranjera en diversos países de Latinoamérica y particularmente aquellos que hacen parte del acuerdo CAN.

Cuando estas empresas generan rentas de fuente extranjera se ven afectadas por el problema de la doble imposición tributaria (pago de tributos de la misma renta en dos Estados), con el fin de evitar este problema y los potenciales de evasión fiscal, Perú y Colombia firmaron el convenio de la Comunidad Andina de Naciones, del cual hacen parte actualmente Bolivia, Colombia, Ecuador y Perú. Por esta razón resulta importante identificar cuáles son las implicancias tributarias para empresas peruanas que obtienen rentas de fuente extranjera generadas en Colombia en aplicación de este convenio.

Según Vega (2003), "el proceso de internacionalización de la vida económica se ha materializado en el fenómeno comúnmente denominado como 
la globalización" (p. 32), el cual implica que las empresas deban competir en un mercado mundial buscando tener presencia en más de un Estado. Al mismo tiempo, "este proceso multiplica los flujos de rentas internacionales y paralelamente, la posibilidad de que se produzca doble imposición internacional" (Vega, 2003, p. 33).

Para evitar que el fenómeno de la doble imposición suponga un obstáculo a la posición competitiva de las empresas globales, es imprescindible desarrollar un proceso de planificación fiscal internacional, dentro de los cuales se encuentran los convenios de doble imposición firmados entre países, con el fin de constituir sistemas jurídicos comunes de coordinación de poderes tributarios para la eliminación de la doble imposición.

En este sentido, señala Taboada (1993):

Es claro que la empresa que consiga reducir su carga fiscal mediante la adecuada planificación fiscal se encontrará en una mejor situación competitiva que otra que, pudiéndolo evitar, soporte una carga fiscal superior (p. 41).

Por consiguiente, los convenios de doble imposición constituyen un medio para eliminar o reducir la imposición en el Estado de la fuente del ingreso. De acuerdo al Departamento de Asuntos Económicos y Sociales de las Naciones Unidas (2013):

Debido a las diferencias que existen entre los diversos regímenes tributarios, los acuerdos fiscales bilaterales son el instrumento más flexible para conciliar regímenes tributarios conflictivos y para evitar o mitigar la doble tributación (p. 139).

\section{Análisis del problema}

Las empresas de los países miembros de la CAN que brindan servicios de consultoría empresarial obtienen beneficios y perjuicios económicos, en el desarrollo de la venta de servicios a otros países miembros del tratado, en este sentido es necesario identificar un conjunto de definiciones básicas 
que enmarcan los criterios del convenio de doble tributación, los cuales se exponen a continuación:

\section{Ingreso de fuente extranjera}

Al prestar el servicio de consultoría contable la empresa peruana reconocerá y revelará en sus estados financieros un ingreso de fuente extranjera, que para efectos de la presentación de su declaración jurada anual de renta debe ser considerado como ingreso no gravado (exonerado) del IR, por lo cual debe ser deducido evitándose así la doble imposición tributaria, y por ende el beneficio tributario a favor de los contribuyentes que generen este tipo de rentas en concordancia con el artículo n. ${ }^{0} 3$ de la decisión n. ${ }^{0}$ 578-CAN. Los demás países miembros que, de conformidad con su legislación interna, se atribuyan potestad de gravar las referidas rentas, deberán considerarlas como exoneradas, para los efectos de la correspondiente determinación del impuesto a la renta o sobre el patrimonio.

Se debe tener en cuenta que este ingreso debe ser reconocido para efectos tributarios según el criterio de lo devengado en mención al inciso a) del artículo n. ${ }^{\circ} 57$ de la legislación peruana sobre el Impuesto de Renta -LIR-, las demás rentas se imputarán al ejercicio gravable en que se perciban, es decir, debe estar efectivamente realizado para su deducción.

\section{Retención de renta de fuente extranjera}

El artículo n. ${ }^{\circ} 14$ de la decisión n. ${ }^{\circ}$ 578-CAN, señala que los ingresos deben ser gravados en el país en cuyo territorio se produzca el beneficio de tales servicios, se presume que es aquel en donde se imputa y registra el correspondiente gasto. En relación a lo mencionado en este artículo, las retenciones de los ingresos percibidos producto de los servicios de consultoría contable deberán ser gravadas en el país donde se imputa y registra el gasto, es decir Colombia. Para tales efectos, las empresas colombianas que reciben los servicios deberán retener el porcentaje de sus rentas según su legislación vigente. Asimismo, deberán entregar a las empresas peruanas 
los certificados de retención del impuesto a la renta correspondiente, para que estas puedan sustentar la retención de rentas ante la administración tributaria del país.

Se debe tener en cuenta que las rentas pagadas en el exterior deben ser reconocidas como crédito al impuesto a la renta según el inciso e del artículo n. ${ }^{\circ} 88$ de la LIR, los impuestos a la renta abonados en el exterior por las rentas de fuente extranjera gravadas por esta ley, siempre que no excedan del importe que resulte de aplicar la tasa media del contribuyente a las rentas obtenidas en el extranjero, ni el impuesto efectivamente pagado en el exterior. El importe que por cualquier circunstancia no se utilice en el ejercicio gravable, no podrá compensarse en otros ejercicios ni dará derecho a devolución alguna, de no poder utilizar dicho crédito, se podrá contabilizar como gasto deducible para el IR según establece el artículo n. ${ }^{\circ}$ 51-A de la LIR, a fin de establecer la renta neta de fuente extranjera, se deducirá de la renta bruta los gastos necesarios para producirla y mantener su fuente.

Sin embargo, la legislación peruana limita la utilización del crédito proveniente de retenciones de fuente extranjera según el cálculo de una tasa media, de acuerdo al inciso e) del artículo n. ${ }^{\circ} 88$ de la LIR y la justificación de su efectivo pago, no permitiendo su compensación en ejercicios posteriores. Argumentando que estas rentas son gravadas en el país de la fuente mas no en Perú, es por ello que no se deben incluir dentro del cálculo de rentas de fuente mundial según nos menciona el inciso b del artículo 37 de la ley del IR. Tampoco podrán incluirse como gasto en el periodo porque está vinculado a un ingreso exonerado para efectos del IR. A fin de establecer la renta neta de tercera categoría se deducirá de la renta bruta los gastos necesarios para producirla y mantener su fuente, así como los vinculados con la generación de ganancias de capital, en tanto la deducción no esté expresamente prohibida por esta ley, en consecuencia son deducibles los tributos que recaen sobre bienes 0 actividades productoras de rentas gravadas.

Mediante este análisis, es posible concluir que las empresas peruanas no obtendrán ningún beneficio fiscal ni económico por las retenciones abonadas en el exterior. 


\section{Gastos inherentes a rentas de fuente extranjera}

Para la generación de ingresos de fuente extranjera las empresas proveedoras de servicios incurren en diversos gastos y costos (desembolsos) contabilizados en sus estados financieros. Al respecto, el artículo n..$^{\circ} 37 \mathrm{de}$ la LIR indica que para hacer deducible estos gastos y costos deben estar vinculados a generar rentas gravadas para efectos de la renta imponible. Así mismo el inciso p del artículo 21 del reglamento de la LIR especifica que cuando los gastos necesarios para producir la renta y mantener la fuente incidan conjuntamente en rentas gravadas, exoneradas o inafectas, y no sean imputables directamente a unas u otras, la deducción se efectuará en forma proporcional al gasto directo imputable a las rentas gravadas.

Además, que en los casos en que no se pudiera establecer la proporcionalidad indicada, se considerará como gasto inherente a la renta gravada el importe que resulte de aplicar al total de los gastos comunes el porcentaje que se obtenga de dividir la renta bruta gravada entre el total de rentas brutas gravadas, exoneradas e inafectas. Por ende, todos los gastos vinculados directamente a los ingresos generados en la prestación del servicio no serán deducibles para el cálculo de la renta neta, lo cual provoca perjuicios económicos, ya que al considerarse estos ingresos como exonerados, según la legislación peruana, deben ser adicionados en la declaración anual del impuesto a la renta.

Cabe señalar, que algunos de estos desembolsos, como por ejemplo los gastos por salarios del contador quien se encarga de llevar la contabilidad de manera global de las transacciones prestadas en Perú y Colombia, no tienen vinculación directa con ingresos exonerados, siendo considerados como gastos comunes para efecto del IR. Por consiguiente, si las empresas tienen registrados gastos comunes vinculados a diversas rentas, estas deberán calcular una adición por gastos inherentes de fuente extranjera (GIFE) tomando en cuenta el siguiente procedimiento mencionado en el artículo n. ${ }^{\circ} 29-\mathrm{B}$ del reglamento de la LIR:

1. Aplicando a dichos gastos el porcentaje que resulte de dividir los gastos directamente imputables a la renta de fuente extranjera a la que se 
encuentre vinculada el gasto, entre la suma de dichos gastos y los gastos directamente imputables a la renta de fuente peruana de la categoría a la que se encuentre vinculada el gasto, multiplicado por cien. El porcentaje se expresará hasta con dos decimales. El monto resultante será el gasto deducible para la determinación de la renta neta de fuente extranjera. El saldo se aplicará para la determinación de la renta neta de fuente peruana de la categoría a la que se encuentre vinculado el gasto, en tanto la ley admita su deducción.

2. En los casos en que no se pudiera establecer dicho porcentaje, se aplicará el siguiente procedimiento:

a. Se sumarán los ingresos netos de las actividades generadoras de renta de fuente extranjera a las que se encuentre vinculado el gasto.

b. El monto obtenido en el inciso a se sumará con los ingresos netos de fuente peruana de la categoría a la que se encuentre vinculada el gasto.

c. El monto obtenido en el inciso a se dividirá entre el obtenido en el inciso b y el resultado se multiplicará por cien. El porcentaje resultante se expresará hasta con dos decimales.

En resumen, las empresas no podrán deducir ningún gasto o costo vinculado directamente a los ingresos de fuente extranjera provenientes de operaciones con países miembros de la CAN, generando un perjuicio económico.

\section{Propuesta: retención del impuesto a la renta sobre el ingreso neto}

El artículo n. ${ }^{\circ} 37$ de la LIR nos indica que para hacer deducible los gastos y costos inherentes al servicio, estos deben estar vinculados a generar rentas gravadas. Pero al aplicar el convenio de la CAN la empresa peruana tiene la obligación de reconocer el ingreso como exonerado del IR, por ende, no 
serán deducibles los gastos y costos vinculados al servicio, disminuyendo así sus beneficios tributarios, ya que en ningún momento deducirá sus gastos.

Es por ello que resulta más beneficioso para las empresas que prestan servicios de consultoría, que la retención de la renta de fuente extranjera se efectúe con base en el ingreso neto, es decir, deduciendo los gastos y costos vinculados al ingreso generado. Lo cual le permite recuperar los beneficios fiscales que por naturaleza de la transacción le corresponden. De ocurrir esto ya no sería necesario considerar como crédito fiscal o gasto a las retenciones.

\section{Conclusiones}

El presente documento permite concluir que las implicancias tributarias de aplicar el convenio de la CAN en la prestación de servicios de consultoría contable empresarial por parte de una empresa peruana a una colombiana en territorio colombiano son:

- La deducción de los ingresos exonerados del impuesto a la renta por parte de la empresa peruana, con el fin de no tributar doble sobre la misma renta.

- La retención y declaración de renta deberá realizarla la empresa colombiana, quien reconoce el servicio como gasto bajo los lineamientos de la decisión n. ${ }^{0} 578$ del convenio de la CAN.

- El tratamiento tributario para determinar el monto deducible de los gastos vinculados a los ingresos del servicio prestado por la empresa peruana de acuerdo a su legislación.

Las empresas de los países miembros de la CAN que brindan servicios de consultoría empresarial obtienen beneficios económicos mediante una reducción de costos tributarios al momento de exportar servicios, ya que evitan el doble pago de IR por sus ingresos obtenidos. 
La empresa colombiana al reconocer el gasto deberá retener el IR correspondiente sobre el ingreso bruto, según lo establecido por el convenio de la CAN, lo cual no será objeto de crédito fiscal ni gasto deducible para la empresa peruana generándole perjuicios económicos y fiscales.

A su vez, esta empresa incurre en gastos y costos inherentes al servicio que no serán deducibles en su declaración anual por provenir de un ingreso de fuente extranjera, que de acuerdo al convenio de la CAN, es considerado exonerado del IR generando perjuicios económicos, con excepción de los gastos comunes, en los que se debe realizar la prorrata de gastos correspondiente.

Las empresas de los países miembros de la CAN que prestan servicios de asesoría deben seguir los lineamientos establecidos por este, para evitar una doble imposición tributaria y posibles conflictos que puedan surgir con la administración tributaria de su país y del país al que se exporta el servicio.

Se recomienda a las empresas que presten servicios de consultoría en el exterior invertir con mayor énfasis en países miembros del convenio CAN, ya que así obtendrán mayores beneficios económicos y fiscales.

Para evitar que la retención del impuesto a la renta sobre el ingreso bruto afecte a las empresas que prestan servicios de consultoría, y en aras de promover la inversión privada y el desarrollo económico-social mediante la prestación de servicios entre países miembros de la CAN, creemos conveniente recomendar que la retención del impuesto a la renta debería darse sobre el ingreso neto percibido por la empresa peruana, mas no sobre el ingreso bruto como se viene realizando en la actualidad. 


\section{Referencias}

Actualidad Empresarial. (2016). Texto único ordenado de la ley del impuesto a la renta. Recuperado de http://aempresarial.com/servicios/pioner/05_2013_7_ ONTBL.pdf

Calderón, J. M. (2004). La incidencia de la globalización en la configuración del ordenamiento tributario del siglo XXI. Madrid: Instituto de Estudios Fiscales.

Departamento de Asuntos Económicos y Sociales de las Naciones Unidas. (2013). Convención modelo de las Naciones Unidas sobre la doble tributación entre países desarrollados y países en desarrollo, revisión de 2011. Nueva York.

Dirección de Impuestos y Aduanas Nacionales. (2016). Decisión 578. Régimen para evitar la doble tributación y prevenir la evasión fiscal. Bogotá. Recuperado de http://www.dian.gov.co/descargas/convocatorias/128_2009/Documentos GuiaNo.2/Decision_CAN_578_04052005.pdf

Gálvez, S. (2010, abril). Tratamiento de los créditos por impuesto a la renta abonados en el exterior. Revista de X Jornadas Nacionales de Tributación de IFA-Perú, (28), 1. Perú.

Superintendencia Nacional de Administración Tributaria y Aduanas. (2016). Informe $n .{ }^{\circ}$ 023-2015-SUNAT/5D0000. Recuperado de http://www.sunat.gob. pe/legislacion/oficios/2015/indcor.htm

Taboada, P. (1993). Planificación fiscal de la inversión española en el extranjero. Primera parte. Perspectivas del Sistema Financiero, (41), 139.

Vega, F. A. (2003). Las cláusulas de limitación de beneficios en los convenios para evitar la doble imposición. Madrid: Instituto de Estudios Fiscales. 


\section{Anexos}

Anexo 1. Matriz de consistencia.

\begin{tabular}{|c|c|c|c|c|}
\hline $\begin{array}{c}\text { Problema } \\
\text { general }\end{array}$ & Objetivo general & Hipótesis general & \multicolumn{2}{|c|}{ Variables } \\
\hline \multirow[b]{2}{*}{$\begin{array}{l}\text { ¿Cuáles son las } \\
\text { implicancias tribu- } \\
\text { tarias de la aplica- } \\
\text { ción del convenio } \\
\text { de la CAN en los } \\
\text { servicios de con- } \\
\text { sultoría contable } \\
\text { prestados por Perú } \\
\text { a Colombia en el } \\
\text { periodo 2015? }\end{array}$} & \multirow[b]{2}{*}{$\begin{array}{l}\text { Identificar las } \\
\text { implicancias tribu- } \\
\text { tarias de la aplica- } \\
\text { ción del convenio } \\
\text { de la CAN en los } \\
\text { servicios de con- } \\
\text { sultoría contable } \\
\text { prestados por Perú } \\
\text { a Colombia en el } \\
\text { periodo } 2015 \text {. }\end{array}$} & \multirow{2}{*}{$\begin{array}{l}\text { Al aplicar el conve- } \\
\text { nio de la CAN, Perú } \\
\text { reconocerá su ingreso } \\
\text { como exonerado } \\
\text { deducible para efectos } \\
\text { del IR, además se } \\
\text { le retendrá el IR } \\
\text { correspondiente, que } \\
\text { no podrá ser usado ni } \\
\text { como crédito fiscal ni } \\
\text { como gasto para efec- } \\
\text { to del IR. Así mismo, } \\
\text { los gastos directa- } \\
\text { mente relacionados } \\
\text { al servicio no serán } \\
\text { deducibles. }\end{array}$} & Dependiente & $\begin{array}{l}\text { Implicancias } \\
\text { tributarias }\end{array}$ \\
\hline & & & Independientes & $\begin{array}{l}\text { Convenio de } \\
\text { la CAN }\end{array}$ \\
\hline $\begin{array}{l}\text { Problema } \\
\text { específico } 1\end{array}$ & $\begin{array}{c}\text { Objetivo } \\
\text { específico } 1\end{array}$ & $\begin{array}{c}\text { Hipótesis } \\
\text { específica } 1\end{array}$ & \multicolumn{2}{|c|}{ Variables } \\
\hline \multirow[b]{2}{*}{$\begin{array}{l}\text { ¿Cuáles son los } \\
\text { beneficios econó- } \\
\text { micos que genera } \\
\text { la aplicación del } \\
\text { convenio de la } \\
\text { CAN en la presta- } \\
\text { ción de servicios } \\
\text { de consultoría } \\
\text { contable entre } \\
\text { Perú y Colombia } \\
\text { en el periodo } \\
2015 ?\end{array}$} & \multirow[b]{2}{*}{$\begin{array}{l}\text { Describir cuáles } \\
\text { son los beneficios } \\
\text { económicos que } \\
\text { genera la aplica- } \\
\text { ción del convenio } \\
\text { de la CAN en la } \\
\text { prestación de ser- } \\
\text { vicios de consulto- } \\
\text { ría contable entre } \\
\text { Perú y Colombia } \\
\text { en el periodo } 2015 \text {. }\end{array}$} & \multirow{2}{*}{$\begin{array}{l}\text { En concordancia con } \\
\text { el artículo n. }{ }^{\circ} 14 \text { de la } \\
\text { decisión n. } 578 \text { del } \\
\text { convenio de la CAN, } \\
\text { las rentas obtenidas } \\
\text { producto de la pres- } \\
\text { tación de servicios de } \\
\text { consultoría serán gra- } \\
\text { vables solo en un país } \\
\text { miembro, evitando así } \\
\text { la doble imposición, } \\
\text { lo cual generaría un } \\
\text { beneficio económico } \\
\text { para las empresas. }\end{array}$} & Dependiente & $\begin{array}{l}\text { Beneficios } \\
\text { económicos }\end{array}$ \\
\hline & & & Independientes & $\begin{array}{l}\text { Convenio de } \\
\text { la CAN }\end{array}$ \\
\hline
\end{tabular}




\begin{tabular}{|c|c|c|c|c|}
\hline $\begin{array}{c}\text { Problema } \\
\text { específico } 2\end{array}$ & $\begin{array}{c}\text { Objetivo } \\
\text { específico } 2\end{array}$ & $\begin{array}{c}\text { Hipótesis específica } \\
2\end{array}$ & \multicolumn{2}{|c|}{ Variables } \\
\hline $\begin{array}{l}\text { ¿Cuáles son los } \\
\text { perjuicios econó- }\end{array}$ & Describir cuáles & $\begin{array}{l}\text { Al prestar servicios de } \\
\text { consultoría contable } \\
\text { bajo el convenio CAN, } \\
\text { la empresa peruana } \\
\text { tendrá }\end{array}$ & Dependiente & $\begin{array}{l}\text { Perjuicios } \\
\text { económicos }\end{array}$ \\
\hline $\begin{array}{l}\text { micos que genera } \\
\text { la aplicación del } \\
\text { convenio de la } \\
\text { CAN en la presta- } \\
\text { ción de servicios } \\
\text { de consultoría } \\
\text { contable entre } \\
\text { Perú y Colombia } \\
\text { en el periodo } \\
2015 ?\end{array}$ & $\begin{array}{l}\text { económicos que } \\
\text { genera la aplica- } \\
\text { ción del convenio } \\
\text { de la CAN en la } \\
\text { prestación de ser- } \\
\text { vicios de consulto- } \\
\text { ría contable entre } \\
\text { Perú y Colombia } \\
\text { en el periodo } 2015 .\end{array}$ & $\begin{array}{l}\text { gastos y costos vincu- } \\
\text { lados al servicio que } \\
\text { no serán deducibles } \\
\text { para efectos del IR, } \\
\text { tal como establece el } \\
\text { inciso "p" del artículo } \\
\text { n. }{ }^{\circ} 21 \text { del Reglamento } \\
\text { de la Ley del IR } \\
\text { (legislación peruana), } \\
\text { lo cual generaría un } \\
\text { perjuicio económico a } \\
\text { las empresas. }\end{array}$ & Independientes & $\begin{array}{l}\text { Convenio de } \\
\text { la CAN }\end{array}$ \\
\hline
\end{tabular}

Fuente: elaboración propia.

Anexo 2. Índice de abreviaturas.

\begin{tabular}{l|l}
\hline CAN & Convenio de la Comunidad Andina de Naciones \\
\hline DJ & Declaración jurada \\
\hline IR & Impuesto a la renta \\
\hline LIR & Ley del impuesto a la renta - Perú \\
\hline RLIR & Reglamento de la ley del impuesto a la renta \\
\hline
\end{tabular}

Fuente: elaboración propia.

\section{Anexo 3. Cálculo de la tasa media.}

Según el inciso d del artículo n. ${ }^{\circ} 52$ de la RLIR, se dispone que por tasa media se entenderá el porcentaje que resulte de relacionar el impuesto determinado con la renta neta de tercera categoría más la renta neta de fuente extranjera. De existir pérdidas de ejercicios anteriores no se restan de la renta neta. 


\section{Tasa media aplicable del impuesto a la renta abonado en el extranjero}

$$
\text { Tasa media }=\frac{\text { Impuesto determinado }}{\mathrm{RN}} \times 100
$$

Donde:

$\mathrm{RN}$ : renta neta empresarial o de tercera categoría (28\% sobre la base imponible) + renta neta de fuente extranjera.

Fuente: elaboración propia. 